\title{
Empowering the physician-patient relationship: The effect of the Internet
}

\author{
Pearl Jacobson, MIS Candidate \\ Faculty of Information Studies, University of Toronto.
}

\begin{abstract}
Objectives: This review aims to explore the relationship between patient empowerment and the physician-patient relationship in the context of the clinical encounter. Difficulties and opportunities presented by patients' use of the Internet are presented from the perspectives of both patients and health care providers. The review explores roles for librarians in facilitating communication and informed decision-making in the clinical consultation.
\end{abstract}

Methods: Medline was searched using the MeSH terms Physician-Patient Relations, Internet /utilization, Power (Psychology) and Information Services. Scholars Portal was searched using the keywords Internet, Empowerment, and Consumer Health Information.

Main Findings: Studies on how the Internet affects patients' experience of empowerment within the clinical encounter have shown mixed outcomes. While patients' desire to use the Internet for health information is increasing, some patients are reluctant to discuss this information in the clinical encounter for fear of challenging the physician's authority. Some physicians express frustration in dealing with patients who search for information online, while others describe the Internet as beneficial.

Conclusions: While searching the Internet is often personally empowering for patients, this sense of empowerment does not necessarily translate into self-efficacy in interactions with health care providers. Whether health information found on the Internet is empowering for patients in clinical consultations appears to depend on the power relations between patients and practitioners, how patients use the information they retrieve, as well as on physicians' affective responses to these patients. Librarians have a role to play in educating health care consumers and mediating the exchange of information between practitioner and patient.

Keywords: physician-patient relationship, Internet, empowerment, health information, health care consumer

\section{Introduction}

There has been considerable discussion of the role of the Internet in facilitating public access to health information. Policy documents from Health Canada's Office of Health and the Information Highway and the Department of Health in the UK suggest that patients who are more informed will experience a greater sense of agency and 
empowerment and will be better able to manage their health. The increasing availability of online medical information has been the result of growing efforts to cut costs of health care by advocating disease prevention and self-management of chronic conditions (Eysenbach 1714). Sources of health information on the Internet include government portals, association websites, privately-sponsored health information supported by direct-to-consumer advertising, and consumer-produced sites such as personal websites, blogs, and online discussion forums. The growth of these accessible and inexpensive online sources of information, compounded by shortages of general practitioners and time constraints experienced by patients during medical consultations, have led to patients' increased reliance on the Internet for information and advice on medical topics (Anderson, Rainey, and Eysenbach 69-72).

Health care consumers' use of the Internet for health information has grown substantially in the past few years. Eighty percent of American Internet users, or some 113 million adults, have searched for information online in 2005 (Fox), an increase from $62 \%$ of Internet users, or 73 million Americans, in 2002 (Fox and Rainie). In one study, between $7 \%$ and $32 \%$ of those using the Internet for health reported that using the Internet affected a decision about health or health care (Baker et al. 2403). Internet users with chronic diseases are more likely than users with acute illnesses to use the Internet to access health-related information (Bansil et al.).

It has become increasingly common for patients to bring information found on the Internet to their visits with health care professionals. One major U.S. household survey found that $50 \%$ of respondents who found health information on the Internet took this information to consultations with their physicians (Murray et al., "Patient Perceptions" 1731). Eighty-five percent of physicians surveyed in one national U.S. survey had experienced a patient bringing Internet information to a visit (Murray et al., "U.S. National").

The emergence of health information on the Internet and other computer applications assisting patients in health-related matters has led to the development of a new field called consumer health informatics. This branch of health informatics studies health consumers' needs, preferences, and uses of computerized information systems and examines the effects these systems have on patients' participation in managing medical concerns (Eysenbach 1713). Replacing "patient" by "consumer" in this context signifies a growing awareness of the role of patient choice and responsibility. Consumer informatics is thought to increase patient self-efficacy, which is the "confidence in remaining independent and being able to make treatment decisions" (Fleisher 261) by increasing "evidence-based patient choice" (Eysenbach and Jadad) and putting more treatment decisions in the hands of patients.

There has been increasing debate about the effects of the Internet revolution on health care and, in particular, on the physician-patient relationship. Because the Internet provides laypersons with access to information that has traditionally only been available to experts, some argue that the Internet is helping health consumers to become more empowered (Johnson and Ramaprasad 23; Laing, Hogg, and Winkelman 189). This 
paper examines the relationship between Internet information and empowerment in the context of the health care encounter. By reviewing the literature on the effects of the Internet on the physician-patient relationship, this work assesses the impact of the Internet in mediating power relations between physicians and patients and altering the way each experiences the clinical encounter. Implications for information professionals are discussed with respect to the changing dynamics of the health care encounter brought about by the use of online health information.

MEDLINE ${ }^{\circledR}$ was searched using the MeSH terms Physician-Patient Relations, Internet /utilization, Power (Psychology) and Information Services. Scholars Portal was searched using the keywords Internet, Empowerment, and Consumer Health Information. Statistics were searched using government websites, E-Stat database, and the Pew Internet Research site. Studies were included if they dealt with the impact of information—specifically the Internet—on physician-patient interaction. Studies examining access to health information and its relationship to patient empowerment, consumer health informatics, shared decision-making, or informed choice were also included. Articles describing electronic medical records, individual web portals, or patient-provider communication tools were excluded.

\section{Information and Empowerment}

Traditionally, patients received information about diagnosis, prognosis and treatment primarily from physicians. Patients from previous generations generally did not challenge physicians' advice, nor did they question prescribed treatments. In this hierarchical model, patients were passive recipients of information that was filtered and dispensed by health care providers. The past ten years have seen a shift away from this model of "benevolent paternalism" (Badcott 174) and towards a model of shared decision-making in which the "informed patient" takes an active role in his or her health care (Kivits 280). Patient engagement in decision-making is increasing due to broader access to information, increased patient autonomy, and patients' ability to acquire the tools to research clinical conditions and interpret medical data (Woolf et al. 293). A revised physician-patient relationship has grown out of this new model of decisionmaking in which the physician recognizes the need of the patient to make an "informed choice" and to participate as a partner in interpreting medical information and selecting treatment options (Eysenbach and Jadad).

It has been argued that access to information is a source of patient empowerment (Broom 325-45; Lupton 373-81). "Patient empowerment" is a term that refers to a patient role in which the distribution of power between physician and patient has been altered in such a way that patients are able to "take charge of their health and their interactions with health care professionals" (Roberts 87). Importantly, two types of patient empowerment are to be distinguished: empowerment in the context of the patient-provider interaction, in which knowledge, values and power are shared; and empowerment from the point of view of the patient, which is seen as a process of personal transformation (Aujoulat, d'Hoore, and Deccache sec. 3.2). Lupton (374) has asserted that the emergence of the informed consumer who is able to analyze and 
assess expert knowledge signifies the notion of a "reflexive actor" who is empowered by his or her sense of agency. The informed patient uses the Internet to find second opinions, interpret symptoms, seek support, help interpret physicians' advice, and formulate questions for subsequent visits (Ziebland 566-7). In doing so, she "breaks down the monopoly of medical knowledge by professionals" (Hardey 401), thereby increasing the amount of control she has over the management of her disease.

There are several constraints on the notion of patients being empowered through increased access to information. Much of the discourse around patient empowerment presupposes that people want to be more educated about health care options. Because of the emotional pressures involved in making complex health decisions, and the cost and time involved in researching treatments, patients may feel more comfortable leaving certain decisions to professionals (MacStravic 30-1; Woolf et al. 294). Many patients lack the critical appraisal skills necessary to understand and interpret medical data, leading to confusion and misinformation. This risk is especially acute on the Internet, since many websites lack clear markers of authorship or credibility (Eysenbach and Jadad).

\section{The Effect of the Internet}

Studies on how the Internet affects patients' experience of empowerment within the clinical encounter have shown mixed outcomes. In a study of people newly diagnosed with cancer, participants reported that getting Internet health information helped them talk to their physicians, made them feel more confident in asking questions, and helped them feel empowered to make decisions (Fleisher 260-4). Patients using a weight-loss drug were better able to manage their condition by recognizing and monitoring symptoms, managing acute episodes, using community resources, and controlling negative emotions and responses to illness (Fox, Ward, and O'Rourke 1303-5). Murray et al. ("Patient Perceptions" 1730) reported that success in finding information on the Internet was associated with being "proactive." Patients felt that they could use the information they found online to propose to physicians that they try the latest, most upto-date treatments (Murray et al., "Patient Perceptions" 1731). In a qualitative study of men with prostate cancer, the use of the Internet allowed respondents to feel that there was "something they could do" (Broom 335) to control the course of their disease. Searching for online health information also allowed them to experience a sense of agency by being able to use the information they retrieved to help others dealing with the same condition (Broom 335).

Despite patients' desire to use the Internet for health information, some patients are reluctant to discuss this information in the clinical encounter. In a study of women seeking information about hormone replacement therapy, participants worried about appearing to over-step the boundary between "expert" and "patient," were reluctant to challenge the doctor's expertise, and were wary of putting unnecessary pressures on the busy practitioner (Henwood et al. 601-2). Another study found that while patients actively searched the Internet at home, once in the doctor's office they "[gave] up the 
searcher attitude" and assumed roles of passive receivers of information (Kivits 279). These patients preferred not to reveal that they used the Internet because of the negative reactions they anticipated receiving from physicians (Kivits 279). These results support the view that while searching the Internet is often personally empowering for patients, this sense of empowerment does not necessarily translate into self-efficacy in interactions with health care providers.

Many patients encountered resistance from physicians to discussing online health information. Some patients noted that when the information they found did not coincide with the physician's views, their GPs "decisively rejected or dismissed" their findings (Henwood et al. 605). Hart, Henwood, and Wyatt reported that some physicians dismissed patients' acquired knowledge in attempts to assert their authority, leading patients to be cautious in challenging their physicians' opinions. Patients' online searching could result in physicians showing hostility or irritation, in some cases labeling persons as "over-informed" or "problem patients" (Broom 336). These types of reactions have been interpreted by Broom (342) as strategies for re-asserting the conventional, hierarchical consultation model in situations where the physician feels that his expertise or authority is threatened.

Some physicians have expressed skepticism or frustration with the use of Internet health information in medical consultations. A study of oncologists found that $54 \%$ of physicians perceived the Internet as having a negative effect on patients and/or the physician-patient relationship (Helft 945). Physicians attributed this effect to the confusion, anxiety, and false hopes generated by patients searching for information online and expressed frustration at having to "re-educate the patient" (Helft 945). Ahmad et al. conducted focus groups with 48 family physicians and found that physicians worried that the Internet was generating patient misinformation, leading to confusion, distress and inappropriate self-treatment. A frequent concern was that low health literacy made it difficult for patients to understand and interpret health messages and that patients were being unwittingly exposed to poorly organized and unreliable sources (Ahmad et al.; Eysenbach and Jadad). Physicians worried about the timeconsuming effort required to contextualize and interpret information for patients (Ahmad et al.; Murray et al., "National U.S."). Other concerns have been inappropriate selfdiagnosis and the Internet's role in feeding the worries of patients with hypochondria (Hart, Henwood and Wyatt). Physicians' lack of familiarity with popular health information sites was another source of frustration (Anderson, Rainey, and Eysenbach 76). This "reversed information gap" was cited as exerting extra pressures on the physician, leading to strain in the doctor-patient interaction (Anderson, Rainey, and Eysenbach 76).

Some physicians described the Internet as having a positive effect. In one large U.S. National survey, $75 \%$ of physicians considered the increase in health information on the Internet to be "a good or very-good thing." Thirty-eight percent of physicians believed that bringing Internet information to the consultation had a "beneficial effect" on the encounter (Murray et al., "U.S. National"). In a study on oncologists' views of Internet use, $36 \%$ of physicians stated that the Internet had a positive effect on their patients 
and/or on the physician-patient relationship. They believed the Internet strengthened the relationship by leading to open discussions about information obtained from the Internet and helping patients feel more in control of their health (Helft 945). Practitioners also noted the Internet's ability to give patients opportunity to have "more equal, intellectual discussions" (Helft 945). Seventy-five percent of physicians in the same study agreed that the Internet increased patients' understanding of their disease, though discussing Internet information was thought to add an average of an extra 10 minutes to a clinical encounter (Helft 946).

Whether health information found on the Internet is empowering for patients appears to depend on patients' personal use of the information they retrieve as well as on physicians' responses to these patients. While searching for online health information helps patients become pro-active and more self-reliant in managing illness, it does not necessarily facilitate communication in the clinical encounter. Patients may be hesitant to appear to "challenge" medical authority by initiating conversations about Internet information. Furthermore, physicians may not be open to dialogue as a result of frustrations and misgivings about the usefulness of online searching. Patients' reports of feeling more empowered, and physicians' reports that the Internet allows them to have discussions with patients on a more equal level, suggests that the Internet is playing a role in altering the traditional power imbalance in the patient-provider relationship, thus significantly affecting the way health care decisions are made and the way patients experience treatment within the clinical encounter.

\section{A Role for Librarians}

The evolving physician-patient relationship suggests an expanding role for librarians and information specialists working in medical settings. Skills in acquiring, organizing, retrieving, and summarizing information are particularly useful in such a role. Homan and McGowan (81) suggest that medical librarians with training in clinical or evidencebased medicine are especially well-suited to working collaboratively with members of a clinical team. Using clinical librarians and accredited "informationists" is one way hospitals and clinics could mitigate the frustrations encountered in the health care encounter and help provide access to quality information for patients and family members (Brown 46-8). Informationists could help patients and families understand medical jargon and work individually with each patient to assess their information needs in the context of a particular illness or diagnosis. This type of service could be delivered directly to patients by attaching articles or web printouts to patient charts, or through an "information prescription form," filled out by a nurse or physician and compiled, summarized, or given value-added features by a librarian (Calabretta 36 ). These functions would complement the clinical librarian's role within the physical library by providing outreach services to patients who might be inclined to do research on the Internet instead of visiting the hospital library.

Another role for librarians is as educators, helping patients and families make sense of the vast amounts of Internet health information and informing consumers about the risks associated with inappropriate information and self-diagnosis. Working in patient 
libraries in health centres or hospitals, librarians acting as "evidence educators" (Homan and McGowan 82) could educate the public on how to appraise, synthesize, and compare medical research within an evidence-based framework. Librarians could also work with hospital website developers on portals designed to assist patients and practitioners in evaluating the quality of medical and health information on the Internet. In addition to training patients on the use of free databases such as PubMed ${ }^{\circledR}$, MEDLINEplus ${ }^{\circledR}$, or Clinical Trials.gov, librarians could play a role in introducing medical students and practitioners to databases and websites of interest to patients. Hollander and Lanier (399) suggest that librarian-educators partner with academic and public libraries to enhance access to reliable electronic sources of health information, such as websites sponsored by government agencies, academic medical centres, or nonprofit organizations. Further roles for librarian-educators include teaching classes on evaluating health information on the Internet and providing Web-based guides and tutorials on how to find, judge, and interpret online sources (Gillaspy 483). Librarians teaching at "Mini-Med Schools" are already using community outreach venues such as public lecture series to enhance knowledge of health issues and improve Internet health information literacy among the public (Van Moorsel 28-9).

Lastly, Homan and McGowan (81) have suggested that librarians could serve as "infomediaries" between the patient and physician, helping patients to take part in shared, informed decisions with health care providers. In this model, the librarian could mediate between the patient's desire to use information sought online and the physician's need to vet this information and interpret it for the patient. Calvano and Needham (256) have suggested a related role which they call the "health intermediary." These practitioners would be somewhat similar to knowledge translators, being primarily responsible for translating medical jargon and helping patients convert health information into useable knowledge. Although Calvano and Needham were writing at a time before the widespread use of the Internet, they indicate that health intermediaries would be particularly beneficial at a time when expanding information technologies are requiring health consumers to make sense of vast amounts of information distributed outside the library context.

\section{Conclusion}

While the Internet has the potential to transform access to health information by increasing patients' sense of satisfaction and empowerment, it may also cause difficulties within the context of the physician-patient encounter. The empowering nature of the Internet is constrained by the ability of the public to comprehend and appraise information found online and by physicians' receptiveness and support of patients who want to be more informed. As physicians' roles change from being the "processors of information rather than the providers of information" (Hart, Henwood and Wyatt), information professionals' roles will need to change to address the increased need for mediation between patients and providers, and to facilitate shared treatment planning. Librarians and health professionals_including nurses, pharmacists, and other members of the health care team-can use the Internet as an opportunity to clarify information and relieve anxiety, thus potentially strengthening rapport with 
patients. Patients' use of the Internet for health information will continue to evolve, empowering both physician and patient and, ultimately, shaping a new orientation to health care based on communication, education and shared knowledge.

\section{Works Cited}

Ahmad, F., et al. "Are Physicians Ready for Patients with Internet-Based Health Information?" Journal of Medical Internet Research 8.3 (2006): 22- . 25 Nov. 2006. <http://www.jmir.org/2006/3/e22/html>

Anderson, J. G., M. R. Rainey, and G. Eysenbach. "The Impact of CyberHealthcare on the Physician-Patient Relationship." Journal of Medical Systems 27.1 (2003): 67-84.

Aujoulat, I., W. d'Hoore, and A. Deccache. "Patient Empowerment in Theory and Practice: Polysemy Or Cacophony?" Patient education and counseling 1 Nov. (2006): Sec. 1-4. PubMed $^{\circledR}$. ScienceDirect. University of Toronto Libraries. 21 Nov. 2006 <http://www.sciencedirect.com/>.

Badcott, D. "The Expert Patient: Valid Recognition Or False Hope?" Medicine, Health Care, and Philosophy 8.2 (2005): 173-8.

Baker, L., et al. "Use of the Internet and e-Mail for Health Care Information: Results from a National Survey." JAMA: the Journal of the American Medical Association 289.18 (2003): 2400-6.

Bansil, P. et al. "Health-related Information on the Web: Results from the HealthStyles Survey, 2002-2003." Preventing Chronic Disease 3.2. (2006). 22 Nov. 2006. <http://www.cdc.gov/PCD/issues/2006/apr/toc.htm> 
Broom, A. "Virtually he@lthy: The Impact of Internet use on Disease Experience and the Doctor-Patient Relationship." Qualitative Health Research 15.3 (2005): 325-45.

Brown, H. "Clinical Medical Librarian to Clinical Informationist." Reference Services Review 32.1 (2004): 45-9.

Calabretta, N. "Consumer-Driven, Patient-Centered Health Care in the Age of Electronic Information." Journal of the Medical Library Association 90.1 (2002): 32-7.

Calvano, M., and G. Needham. "Public Empowerment through Accessible Health Information." Bulletin of the Medical Library Association 84.2 (1996): 253-6.

Eysenbach, G., and A. R. Jadad. "Evidence-Based Patient Choice and Consumer Health Informatics in the Internet Age." Journal of Medical Internet Research 3.2 (2001): 19- . 25 Nov. 2006. <http://www.jmir.org/2001/2/e19/html>

Eysenbach, G. "Consumer Health Informatics." BMJ 320.7251 (2000): 1713-6.

Fleisher, L., et al. "Relationships among Internet Health Information use, Patient Behavior and Self Efficacy in Newly Diagnosed Cancer Patients Who Contact the National Cancer Institute's NCI Atlantic Region Cancer Information Service (CIS)." Proceedings of the American Medical Informatics Association Annual Symposium, 260-264. 2002. 23 Nov. 2006. <http://www.amia.org/pubs/proceedings/symposia/2002/053.pdf> 
Fox, S. "Online Health Search 2006." The Pew Internet and American Life Project. 29 Oct. 2006. 20 Nov. 2006. <http://www.pewinternet.org/PPF/r/190/report display.asp>

Fox, S., \& Rainie, L. "Vital Decisions." The Pew Internet and American Life Project. 22 May 2002. 20 Nov. 2006. <http://www.pewinternet.org/PPF/r/59/report display.asp>

Fox, N. J., K. J. Ward, and A. J. O'Rourke. "The 'Expert Patient': Empowerment or Medical Dominance? the Case of Weight Loss, Pharmaceutical Drugs and the Internet." Social Science \& Medicine 60.6 (2005): 1299-1309.

Gillaspy, M. L. "Factors Affecting the Provision of Consumer Health Information in Public Libraries: The Last Five Years." Library Trends 53.3 (2005): 480-95.

Hardey, M. "'E-Health': The Internet and the Transformation of Patients into Consumers and Producers of Health Knowledge." Information, Communication \& Society 4.3 (2001): 388-405.

Hart, A., Henwood, F., and S. Wyatt. The role of the Internet in patient-practitioner relationships: findings from a qualitative research study. Journal of Medical Internet Research 6.3 (2004): 36- . 25 Nov. 2006. <http://www.jmir.org/2004/3/e36/html>

Helft, P. R., F. Hlubocky, and C. K. Daugherty. "American Oncologists' Views of Internet use by Cancer Patients: A Mail Survey of American Society of Clinical Oncology Members." Journal of Clinical Oncology 21.5 (2003): 942-7. 
Henwood, F., et al. "'Ignorance is Bliss Sometimes': Constraints on the Emergence of the 'Informed Patient' in the Changing Landscapes of Health Information." Sociology of health \& Illness 25.6 (2003): 589-607.

Hollander, S., and D. Lanier. "The Physician-Patient Relationship in an Electronic Environment: A Regional Snapshot." Bulletin of the Medical Library Association 89.4 (2001): 397-9.

Homan, J. M., and J. J. McGowan. "The Medical Library Association: Promoting New Roles for Health Information Professionals." Journal of the Medical Library Association 90.1 (2002): 80-5.

Johnson, G. L., and A. Ramaprasad. "Patient-Physician Relationships in the Information Age." Marketing Health Services 20.1 (2000): 20-7.

Kivits, J. "Informed Patients and the Internet: A Mediated Context for Consultations with Health Professionals." Journal of Health Psychology 11.2 (2006): 269-82.

Laing, A., G. Hogg, and D. Winkelman. "Healthcare and the Information Revolution: ReConfiguring the Healthcare Service Encounter." Health Services Management Research 17.3 (2004): 188-99.

Lupton, D. "Consumerism, Reflexivity and the Medical Encounter." Social Science \& Medicine 45.3 (1997): 373-81.

MacStravic, S. "The Downside of Patient Empowerment." Health Forum Journal 43.1 (2000): 30-1. 
Murray, E., et al. "The Impact of Health Information on the Internet on Health Care and the Physician-Patient Relationship: National U.S. Survey among 1.050 U.S. Physicians." Journal of Medical Internet Research 5.3 (2003): 17- . 25 Nov. 2006. <http://www.jmir.org/2003/3/e17/html>

Murray, E., et al. "The Impact of Health Information on the Internet on the PhysicianPatient Relationship: Patient Perceptions." Archives of Internal Medicine 163.14 (2003): 1727-34.

Office of Health and the Information Highway, Health Canada. Canada Health Infoway: Paths to Better Health. Final Report of the Advisory Council on Health Infostructure. Ottawa: OHIH, 1999. 22 Nov. 2006. <http://www.hc-sc.gc.ca/hcs-sss/pubs/ehealthesante/1999-paths-voies-fin/index e.html>

Roberts, K. J. "Patient Empowerment in the United States: A Critical Commentary." Health Expectations: An International Journal of Public Participation in Health Care and Health Policy 2.2 (1999): 82-92.

Van Moorsel, G. "Do You Mini-Med School? Leveraging Library Resources to Improve Internet Consumer Health Information Literacy." Medical Reference Services Quarterly 20.4 (2001): 27-37.

Woolf, S. H., et al. "Promoting Informed Choice: Transforming Health Care to Dispense Knowledge for Decision Making." Annals of Internal Medicine 143.4 (2005): 293300. 
Partnership: the Canadian Journal of Library and Information Practice and Research, vol. 2, no. 1 (2007)

Ziebland, S., et al. "How the Internet Affects Patients' Experience of Cancer: A Qualitative Study." BMJ 328.7439 (2004): 564-569. 